ESAIM: COCV

Vol. 13, No 2, 2007, pp. 413-417

DOI: $10.1051 /$ cocv:2007019
ESAIM: Control, Optimisation and Calculus of Variations

www.edpsciences.org/cocv

\title{
REGULARITY AND VARIATIONALITY OF SOLUTIONS TO HAMILTON-JACOBI EQUATIONS. \\ PART I: REGULARITY \\ (ERRATA)
}

\author{
Andrea C. G. MennucCi ${ }^{1}$
}

\begin{abstract}
This errata corrects one error in the 2004 version of this paper [Mennucci, ESAIM: COCV 10 (2004) 426-451].
\end{abstract}

Mathematics Subject Classification. 49L25, 53C22, 53C60.

Received October 24, 2006.

After the publication of [7] in 2004, it became clear that the regularity of the form $\alpha$ in Lemma 4.4 had to be related to the regularity of $K$ and of $u_{0}$; this influences the minimal regularity of $K, u_{0}$, as needed in hypotheses in Lemma 4.4, in Theorem 4.1, and in many following relevant discussions. This errata corrects that error; to keep the matter short, all material that is unaffected by the error is omitted; whereas care was taken so that results and discussions that are here corrected retain the original numbering as in [7].

\subsection{Regularity of conjugate points}

We will prove in this section results regarding the set of focal points; each following result extends to the set $\Gamma$ of conjugate points that is a subset of the focal points.

Theorem 4.1. Assume $(\mathrm{CC} 0, \mathrm{H} 1, \mathrm{H} 2)$. If $u_{0}, K, H$ are regular enough, then, by Lemma 4.4 , there is a (at most) countable number of $n-1$ dimensional submanifolds of $\mathbb{R} \times O$ that cover all the sets $G^{i}$; these submanifolds are graphs of functions $\lambda_{i, h}: A_{i, h} \rightarrow \mathbb{R}$ (for $h=1 \ldots$ ) where $A_{i, h} \subset O$ are open sets. The least regular case is $i=n-1$, and the regularity of the $\lambda$ functions is related to the regularity of $u_{0}, K, H$, and to the dimension $\operatorname{dim}(M)=n$ as in the following table:

\begin{tabular}{l|l|l|l}
$\operatorname{dim}(M)$ & $u_{0}, K$ & $H$ & $\lambda$ \\
\hline$n=2$ & $C^{(R+2, \theta)}$ & $C^{(R+2, \theta)}$ & $C^{(R, \theta)}$ \\
$n \geq 3$ & $C^{(R+2, \theta)}$ & $C^{(R+n-1, \theta)} \cap C^{n}$ & $C^{(R, \theta)}$
\end{tabular}

where $R \in \mathbb{N}, \theta \in[0,1]$.

Keywords and phrases. Hamilton-Jacobi equations, cutlocus, conjugate points.

1 Scuola Normale Superiore, Piazza dei Cavalieri 7, 56126 Pisa, Italy; a.mennucci@sns.it 
We now infer some explanatory results on the regularity of the focal points $X\left(\cup_{i} G^{i}\right)$ from the above theorem.

At the lowest regularity, when $u_{0}, K \in C^{2}, H \in C^{n}$, we know that $X \in C^{1}$ and that the sets $G^{i}$ are graphs; we conclude that the set of focal points has measure zero. When $u_{0}, K \in C^{(2, \theta)}, H \in C^{n} \cap C^{(2, \theta)}$, we know that the dimension of the sets $G^{i}$ does not exceed $n-\theta$; so again we conclude that the set of focal points has dimension at most $n-\theta$. In the case $\theta=1$, we can obtain the set of all focal points is rectifiable; that is, if $u_{0}, K \in C^{(2,1)}, H \in C^{n} \cap C^{(2,1)}$, then the sets $G^{i}$ are covered by Lipshitz graphs, so (by known results in [2]) the set of focal points may be covered by $(n-1)$-dimensional $C^{1}$ regular submanifolds of $M$, but for a set of Hausdorff $\mathcal{H}^{n-1}$ measure zero.

When we further raise the regularity, we may suppose that $u_{0}, K \in C^{s+3}, H \in C^{s+n}$ (with $\left.s \in \mathbb{N}\right)^{1}$; then the sets $G^{i}$ are covered by graphs $(\lambda(y), y)$ inside $\mathbb{R} \times O$ of regularity $C^{1+s}$; while $X \in C^{2+s}$ (at least), and we restrict it to those graphs; we can then apply Theorem A.4 to state that the focal points are covered by $C^{1+s}$ regular submanifolds of $M$ but for a set of $\mathcal{H}^{\alpha}$ measure zero, where $\alpha \doteq n-2+1 /(1+s)$.

[... unchanged material deleted...]

The main tool is this lemma; the complete proof of the lemma is in Section 6 .

Lemma 4.4. We assume that the hypotheses $(\mathrm{CC} 0, \mathrm{H} 1, \mathrm{H} 2)$ hold.

We set the regularity of the data $u_{0}, K, H$ by defining parameters $R, R^{\prime} \in \mathbb{N}, \theta, \theta^{\prime} \in[0,1]$, and assuming that

$$
u_{0} \in C^{\left(R^{\prime}+2, \theta^{\prime}\right)}, \quad K \in C^{\left(R^{\prime}+2, \theta^{\prime}\right)}, \quad H \in C^{(R+2, \theta)} ;
$$

by Proposition 3.7, the flow $\Phi=(X, P)$ is $C^{(R+1, \theta)}$ regular; and $O$ is a $C^{\left(R^{\prime}+1, \theta^{\prime}\right)} \cup C^{(R+2, \theta)}$ manifold (that is, the least regular of the two).

Lets fix $i \geq 1, i \leq n-1$, and fix a point $\left(s^{\prime}, y^{\prime}\right) \in \mathbb{R} \times O$, such that $\left(s^{\prime}, y^{\prime}\right) \in G^{(i)}$.

Let $\mathcal{U}$ be a neighbourhood of 0 in $\mathbb{R}^{n-1}$ and let $\phi: \mathcal{U} \rightarrow O$ be a local chart to the neighbourhood $\phi(\mathcal{U})$ of $y^{\prime}=\phi(0)$. The map $\phi$ has regularity $C^{\left(R^{\prime}+1, \theta^{\prime}\right)} \cup C^{(R+2, \theta)}$. In the following, $y$ will be a point in $\phi(\mathcal{U})$.

To study $G^{(i)}$, we should study the rank of the Jacobian of the map $(t, x) \mapsto X(t, \phi(x))$; since the regularity of $X$ is related only to the regularity of $H$, it will be useful to decouple this Jacobian in two parts. To this end, we define a $n$-form $\alpha$ on $\mathbb{R} \times O$, with requirement that $\alpha(t, y)=\alpha(y)$ (that is, $\alpha$ does not depend on $t$ ).

Writing $X^{(t, y)}$ for $X(t, y)$, let

$$
X^{(t, y)^{*}} \alpha
$$

be the push-forward of $\alpha$ along $X ; X^{(t, y)^{*}} \alpha$ is then a tangent form defined on $T_{X(t, y)} M$; it will be precisely defined in equation (6.2). We remark that $X^{(t, y)^{*}} \alpha=0$ iff $(t, y) \in \bigcup_{j} G^{j}$. Note that the pushforward $X^{(t, y)^{*}}$ is $C^{(R, \theta)}$ regular, while the form $\alpha$ is as regular as TO, that is, $\alpha$ is $C^{\left(R^{\prime}, \theta^{\prime}\right)} \cup C^{(R+1, \theta)}$.

Note that, since $X$ solves an O.D.E., then $X$ and $\frac{\partial}{\partial t} X$ have the same regularity; note moreover that

$$
\frac{\partial^{j}}{\partial t^{j}}\left(X^{\left(s^{\prime}, y^{\prime}\right)^{*}} \alpha\right)=\left(\frac{\partial^{j}}{\partial t^{j}} X\right)^{\left(s^{\prime}, y^{\prime}\right)^{*}} \alpha
$$

since $\alpha$ does not depend on $t$. So, by hypotheses and by the definition (6.2) of $X^{(t, y)^{*}} \alpha$, the forms $X^{(t, y)^{*}} \alpha$ and $\frac{\partial}{\partial t}\left(X^{(t, y)^{*}} \alpha\right.$ ) have regularity $C^{(R, \theta)} \cap C^{\left(R^{\prime}, \theta^{\prime}\right)}$ (see also Eq. (6.3)); the derivates $\frac{\partial^{j}}{\partial t^{j}} X^{\left(s^{\prime}, y^{\prime}\right)^{*}} \alpha$ with $j \geq 1$ have regularity $C^{(R-j+1, \theta)} \cup C^{\left(R^{\prime}, \theta^{\prime}\right)}$.

Then, when $R+1 \geq i$, we prove (in Sect. 6 ) that

$$
X^{\left(s^{\prime}, y^{\prime}\right)^{*}} \alpha=0, \quad \frac{\partial}{\partial t} X^{\left(s^{\prime}, y^{\prime}\right)^{*}} \alpha=0, \quad \cdots \frac{\partial^{i-1}}{\partial t^{i-1}} X^{\left(s^{\prime}, y^{\prime}\right)^{*}} \alpha=0
$$

\footnotetext{
${ }^{1}$ A similar result may be obtained when $u_{0}, K \in C^{(s+3, \theta)}, H \in C^{(s+n, \theta)}$.
} 
whereas

$$
\frac{\partial^{i}}{\partial t^{i}} X^{\left(s^{\prime}, y^{\prime}\right)^{*}} \alpha \neq 0 .
$$

We define eventually the map $F: \mathbb{R} \times \mathbb{R}^{n-1} \rightarrow \mathbb{R}$ given by

$$
F(t, x)=\frac{\partial^{i-1}}{\partial t^{i-1}} X^{(t, \phi(x))^{*}} \alpha
$$

since

$$
\frac{\partial}{\partial t} F(t, x) \stackrel{\text { def }}{=} \frac{\partial^{i}}{\partial t^{i}} X^{(t, \phi(x))^{*}} \alpha \neq 0
$$

the above Dini lemma implies that the set $G^{(i)}$ is locally covered by the graph of a function $\lambda_{i}$ defined on a open subset of $O$; $\lambda$ has the same regularity of $F$, so, if $i=1$ then $\lambda$ is in $C^{R, \theta} \cup C^{\left(R^{\prime}, \theta^{\prime}\right)}$ while for $i \geq 2$ it is $C^{(R-i+2, \theta)} \cup C^{\left(R^{\prime}, \theta^{\prime}\right)}$.

The above directly implies Theorem 4.1.

$[\ldots$ all other results are unchanged ... ]

\section{Applications}

\subsection{The Cauchy problem}

We show now how the above theorems may be used for the Cauchy problem (1.2)

$$
\begin{cases}\frac{\partial}{\partial t} w\left(t, x^{\prime}\right)+H^{\prime}\left(t, x^{\prime}, \frac{\partial}{\partial x^{\prime}} w\left(t, x^{\prime}\right)\right)=0 & \text { for } t>0, x^{\prime} \in M^{\prime} \\ w\left(0, x^{\prime}\right)=w_{0}\left(x^{\prime}\right) & \forall x^{\prime} \in M^{\prime} .\end{cases}
$$

[...the preliminary discussion is unchanged ...]

This improves the results of 4.10, 4.12 and 4.17 in [1]; to provide for an easy comparison, we summarize these results

- if $n^{\prime}=\operatorname{dim}\left(M^{\prime}\right), n=n^{\prime}+1$, if $H^{\prime} \in C^{s}$ with $s=n \vee 3$ and $w_{0} \in C^{2}$, then the set $\Gamma$ has measure zero, so the set $\bar{\Sigma}_{u}=\Sigma \cup \Gamma$ has measure zero;

- if $H, w_{0} \in C^{(2,1)}$, then the set $\Gamma$ is rectifiable, so the set $\bar{\Sigma}_{u}=\Sigma \cup \Gamma$ is rectifiable;

- and when $H^{\prime} \in C^{R+1, \theta}, w_{0} \in C^{R+1, \theta}, R \geq 2, w$ is continuous, we prove that the Hausdorff dimension of $\Gamma \backslash \Sigma$ is at most $\beta$, and moreover $\mathcal{H}^{\beta}(\Gamma \backslash \Sigma)=0$ if $\theta=0$, where $\beta=n^{\prime}-1+2 /(R+\theta)$.

In the counterexample in Section 4.4 in [1], $w_{0}$ is $C^{1,1}\left(M^{\prime}\right)$ and not $C^{2}\left(M^{\prime}\right)$; so our results close the gap between the counterexample, where $w_{0}$ is $C^{1,1}\left(M^{\prime}\right)$, and the theorem, where $w_{0}$ is $C^{2}\left(M^{\prime}\right)$; and actually, studying the counterexample, it is quite clear that, if $w_{0}$ is smoothed to become a $C^{2}\left(M^{\prime}\right)$ function, then the counterexample would not work.

\subsection{Eikonal equation and cutlocus}

As in Section 3.5, consider a smooth Riemannian manifold $M$, and a closed set $K \subset M$ and let $d_{K}(x)=$ $d(x, K)$ be the distance to $K$. We set $u_{0}=0$ : then $O$ is the bundle of unit covectors that are normal to $T K$, and $d_{K}(x)$ coincides with the min solution $u(x)$.

We define

$$
\Sigma_{d_{K}} \stackrel{\text { def }}{=}\left\{x \mid \nexists \nabla d_{K}(x)\right\}
$$

If $K$ is $C^{1}$, then $\Sigma_{d_{K}}$ coincides with $\Sigma$ as defined in (4.1).

Since $d_{K}$ is semiconcave in $M \backslash K, \Sigma_{d_{K}}$ is always rectifiable.

This primal problem is a good test bed to discuss the differences and synergies of the results in this paper and the results in Itoh and Tanaka [4] and Li and Nirenberg [5]. 
- In the example in Section 3 in [6], there is a curve $K \subset \mathbb{R}^{2}, K \in C^{1,1}$ such that $\bar{\Sigma}_{d_{K}}$ has positive Lebesgue measure. Note that in this example $\bar{\Sigma}_{d_{K}} \neq \operatorname{Cut}(K)=\Sigma_{d_{K}}$, so the cutlocus $\operatorname{Cut}(K)$ is rectifiable (but not closed).

We do not know if there is a curve $K \in C^{1,1}$ such that $\operatorname{Cut}(K)$ is not rectifiable. (We recall that, by Prop. 14 in [3], $\operatorname{Cut}(K)$ has always measure zero).

- Theorem 4.1 states that if $K$ is $C^{2}$, then $\Gamma$ has measure zero, so by (1.4) and 4.11.4, we obtain that $\bar{\Sigma}_{d_{K}}=$ $\operatorname{Cut}(K)$ has measure zero; so Theorem 4.1 closes the gap between the counterexample in Section $3[6]$ and the previous available results.

- In example in Remark 1.1 in [5], for all $\theta \in(0,1)$ there is a compact curve $K \in C^{2, \theta}$ such that the distance to the cutlocus is not locally Lipschitz; by Theorem 4.1, the cutlocus has dimension at most $n-\theta$.

We do not know if there exists an example of a compact curve $K \in C^{2, \theta}$ such that $\mathcal{H}^{n-1}(\operatorname{Cut}(K))=\infty$

- By the results in Itoh and Tanaka [4] and Li and Nirenberg [5], when $K \in C^{3}$, the distance to the cutlocus is locally Lipschitz and the cutlocus is rectifiable, and moreover (by Cor 1.1 in [5]), for any $B$ bounded $\mathcal{H}^{n-1}(\operatorname{Cut}(K) \cap B)<\infty$. By Theorem 4.1, the set of (non optimal) focal points is rectifiable as well.

\subsubsection{Improvements}

$[\ldots$ the discussion is unchanged ...]

Corollary 5.1. Consider a 2-dimensional smooth Riemannian manifold $M$; suppose that $K$ is a compact $C^{3+s}$ embedded submanifold.

Then, for any open bounded set $A \subset M$, the set $A \cap \Gamma$ is $C^{s+1}-M^{1 /(s+1)}$-rectifiable: that is, it can be covered by at most countably many $C^{s+1}$ curves, but for a set $E$ such that $\mathcal{M}^{1 /(s+1)}(E)=0$.

\section{Proof of 4.4}

[...the two lemma are unchanged...]

Now we prove Lemma 4.4 .

We want to define the $n$ form $\alpha$ so that $\alpha$ does not depend on $t$; and so that $\alpha=e_{1} \wedge \cdots \wedge e_{n}$ where the vectors fields $e_{n-i+1} \ldots e_{n}$ span the kernel of $\frac{\partial}{\partial \bar{x}} X$ at the point $\left(s^{\prime}, y^{\prime}\right)$ (kernel that we will call $V$ ) while $\frac{\partial}{\partial \bar{x}} X$ is full rank on $e_{1} \ldots e_{n-i}$ (that generate the space $W$ ).

One possible way to this is to fix the local chart $\phi: \mathcal{U} \subset \mathbb{R}^{n-1} \rightarrow O$, define

$$
\hat{e}_{1} \stackrel{\text { def }}{=} \phi^{*} \frac{\partial}{\partial x_{1}}, \ldots \hat{e}_{n-1} \stackrel{\text { def }}{=} \phi^{*} \frac{\partial}{\partial x_{n-1}}, \hat{e}_{n} \stackrel{\text { def }}{=} \frac{\partial}{\partial t}
$$

and then choose a $n \times n$ constant matrix $A$, so that

$$
e_{h} \stackrel{\text { def }}{=} \sum_{k} A_{h, k} \hat{e}_{k}
$$

satisfy the requirements.

$[\ldots$ the rest of the proof is unchanged...]

Acknowledgements. The author thanks Prof. Graziano Crasta for spotting the error that is corrected in this errata. 


\section{REFERENCES}

[1] P. Cannarsa, A. Mennucci and C. Sinestrari, Regularity results for solutions of a class of Hamilton-Jacobi equations. Arch. Rat. Mech. 140 (1997) 197-223 (or preprint 13-95, Dip. Mat., Univ. Tor Vergata, Roma).

[2] H. Federer, Geometric measure theory. Springer-Verlag (1969).

[3] G.J. Galloway, P.T. Chruściel, J.H.G. Fu and R. Howard, On fine differentiability properties of horizons and applications to Riemannian geometry. J. Geom. Phys. 41 (2002) 1-12.

[4] J. Itoh and M. Tanaka, The Lipschitz continuity of the distance function to the cut locus. Trans. AMS 353 (2000) 21-40.

[5] Y.Y. Li and L. Nirenberg, The distance function to the boundary, Finsler geometry and the singular set of viscosity solutions of some Hamilton-Jacobi equations. Comm. Pure Appl. Math. 58 (2005) 85-146 (first received as a personal communication in June 2003).

[6] C. Mantegazza and A.C. Mennucci, Hamilton-Jacobi equations and distance functions on Riemannian manifolds. Appl. Math. Optim. 47 (2002) 1-25.

[7] A.C.G. Mennucci, Regularity and variationality of solutions to Hamilton-Jacobi equations. Part I: regularity. ESAIM: COCV 10 (2004) 426-451. 